\title{
Optimal management of Brugada syndrome
}

\author{
Cristian Stătescu, ${ }^{1,2}$, Teodor Vasilcu, ${ }^{1,2}$, loana Mădălina Chiorescu*,1,2, Grigore \\ Tinică $^{1,2}$, Cătălina Arsenescu-Georgescu ${ }^{1,2}$, Radu Sascău $u^{1,2}$
}

1"Grigore T. Popa" University of Medicine and Pharmacy, lasi, Romania; ${ }^{2 “}$ Prof. Dr. George I.M. Georgescu" Institute of Cardiovascular Diseases, lasi, Romania

\begin{abstract}
Brugada syndrome can induce malignant ventricular arrhythmias in the absence of structural heart disease. The hallmark of the condition is the "coved" (type I) or "saddle-back" (types II and III) ST segment elevation in the right precordial leads. The definite diagnosis requires the documentation of the type I morphology. The electrocardiographic pattern is often dynamic, but it can be unmasked by sodium channel blockers such as flecainide. We report the case of a 33-year old male, with family history significant for sudden cardiac death, who underwent successful cardio-pulmonary resuscitation for ventricular fibrillation-associated cardiac arrest. The 12 lead electrocardiogram performed after the resuscitation maneuvers showed intermittent type 1 Brugada pattern. General physical examination and routine laboratory evaluation were unremarkable. A repeated electrocardiogram revealed sinus tachycardia with right bundle branch block. We performed a flecainide challenge test which reproduced the initial coved-type ST segment elevation in V1 and V2. We decided to implant a ventricular single chamber cardioverter-defibrillator with one therapeutical window for ventricular fibrillation (at $300 \mathrm{~ms}$ ), high energy shocks without antitachycardia pacing. The device was successful in preventing another episode of ventricular fibrillation just 2 days after the procedure. Implantable cardioverter-defibrillators are the most effective secondary prophylaxis therapeutic options for individuals with Brugada Syndrome, but they subject the patient to complications related to device implantation and inappropriate shocks.
\end{abstract}

Keywords: Brugada syndrome, implantable cardioverter defibrillator, flecainide test, ventricular fibrillation

\section{Introduction}

Brugada syndrome $(\mathrm{BrS})$ is a genetic condition that accounts for 1 out of 5 of the sudden cardiac deaths (SCD) caused by malignant ventricular arrhythmias, in the absence of structural heart disease [1]. The syndrome was first reported in 1992 by Brugada and Brugada as a persistent ST segment elevation associated with right bundle branch block [2].

Although a high percentage of patients remain asymptomatic, others experience

Received: February 2017; Accepted after review: May 2017; Published: June 2017.

${ }^{*}$ Corresponding author: Chiorescu Madalina, MD, "Grigore T. Popa" University of Medicine and Pharmacy, 16, Universitatii Street, 700115, lasi, Romania.

E-mail: madalina.chiorescu@gmail.com syncope or (aborted) SCD. The presence of ST segment elevation in the right precordial leads remains the hallmark of the condition and helps distinguish three types of BrS [3]. The electrocardiographic (ECG) morphology of $\mathrm{BrS}$ is intermittent, and it can be unmasked by certain drugs [3], fever [4], hypothermia [5], cocaine and alcohol consumption [6] or inhalation of gasoline vapors [7].

This case report underlines the importance of implantable cardioverter-defibrillators (ICDs) in the secondary prevention of sudden cardiac death of patients with Brugada syndrome. 


\section{Case report}

We present the case of a 33-year old male patient with no relevant personal medical history but with family history significant for sudden cardiac death (a brother at the age of 20). The patient was an emergency admission for syncope and cardiac arrest caused by ventricular fibrillation (VF) after strenuous physical exercise. The patient had presented fever $\left(38^{\circ}\right.$ Celsius) 5 days prior to admission.

After successful cardiopulmonary resuscitation maneuvers and electrical defibrillation, the 12-lead electrocardiogram showed intermittent type 1 Brugada pattern and paroxysmal atrial fibrillation (Figure 1).
General and cardiovascular physical examinations were unremarkable. Routine laboratory evaluation that included comprehensive metabolic panel, complete blood count and blood clotting tests showed mild dyslipidemia and slightly elevated white blood count. A repeated 12-lead electrocardiogram revealed sinus tachycardia with incomplete right bundle branch block. Echocardiogram (performed after resuscitation maneuvers) showed a mildly reduced ejection fraction (44\%). He had normal chest radiography and no ventricular arrhythmias were detected during the twenty-four hour Holter monitoring. Serial ECGs did not reveal type 1 Brugada pattern.

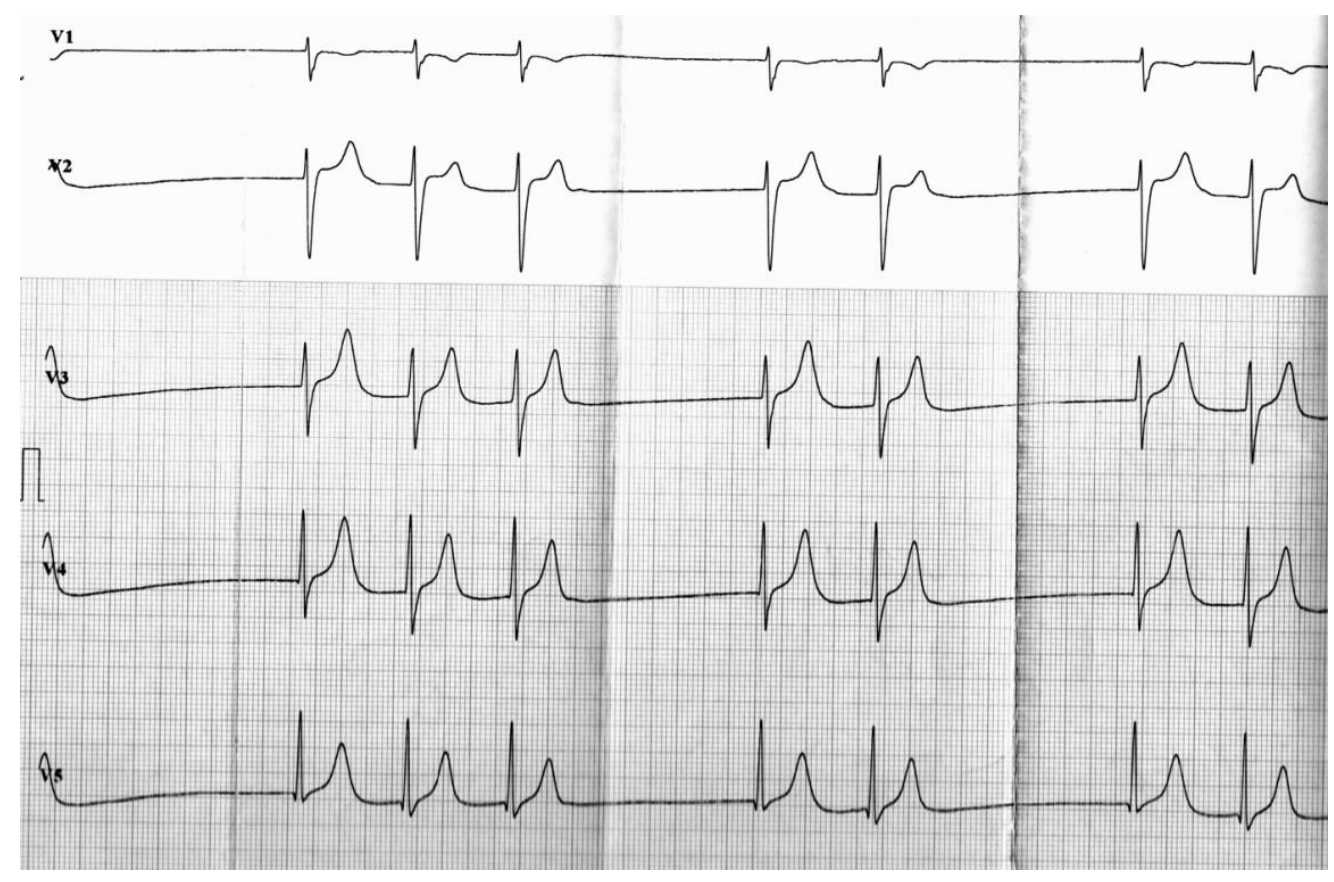

Fig. 1. ECG documenting atrial fibrillation

Because of the dynamic character of the electrocardiographic aspect we performed a flecainide challenge test in order to reproduce the diagnostic pattern of Brugada. The patient was administered $1 \mathrm{mg} / \mathrm{kg}$ intravenous flecainide over 5 minutes, under close monitoring of vital signs. The 12-lead ECG (with the V1 and V2 electrodes placed in the second intercostal space) showed a 2-3 mm coved-type ST segment elevation with a descending negative $T$ wave in the first 2 precordial derivations (Figures 2 and 3). As the flecainide test was positive for type 1
Brugada pattern we performed implantation of a ventricular single chamber (VVI) implantable cardioverter-defibrillator (ICD) with one therapeutical window for ventricular fibrillation (at $300 \mathrm{~ms}$ ), high energy shocks without antitachycardia pacing. The ICD was programed for ventricular backup pacing at $40 / \mathrm{min}$, stimulation threshold was set at 3.5 $\mathrm{mV}$, stimulus duration at $0.4 \mathrm{~ms}$, and sensing threshold at $0.3 \mathrm{mV}$. The procedure was completed successfully, without any local or systemic complications. 


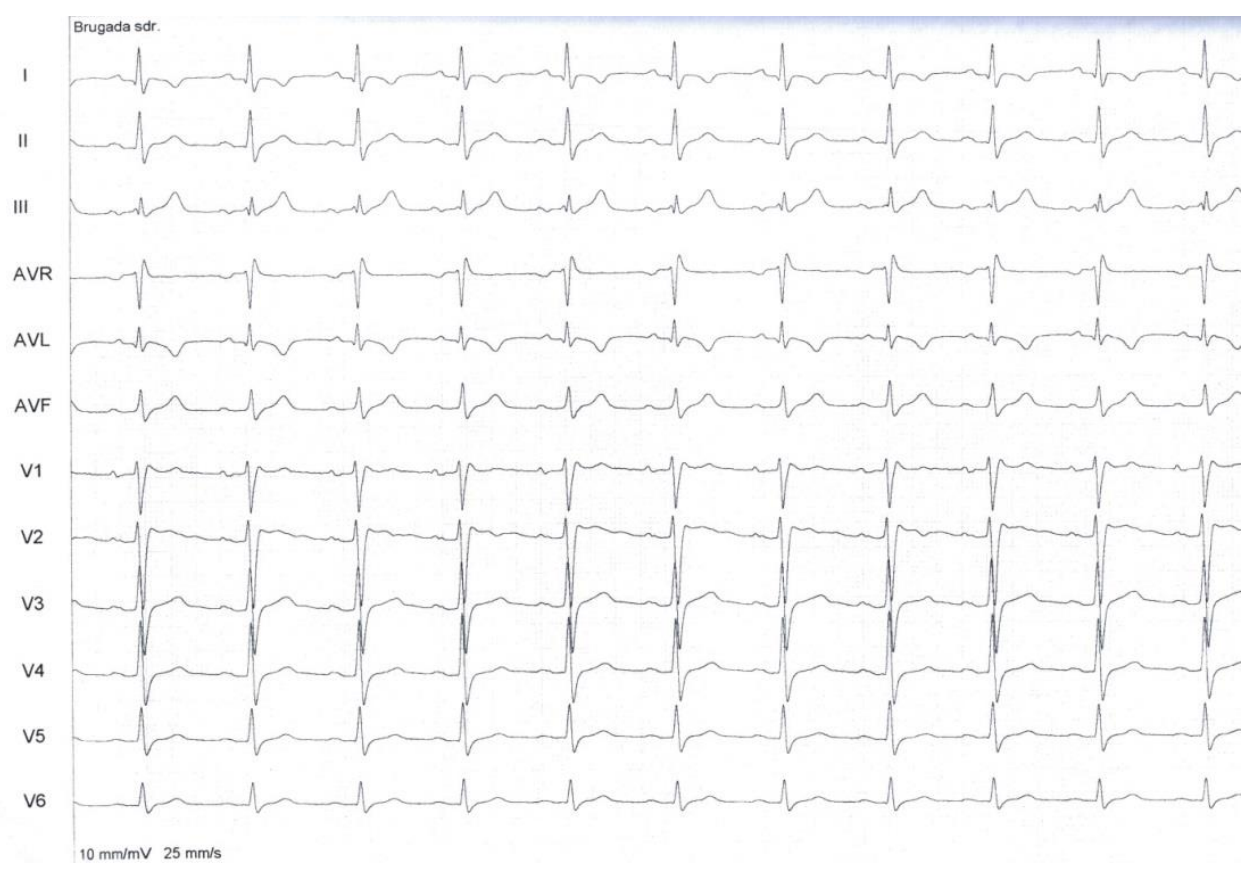

Fig. 2. 12 lead ECG performed before the flecainide test

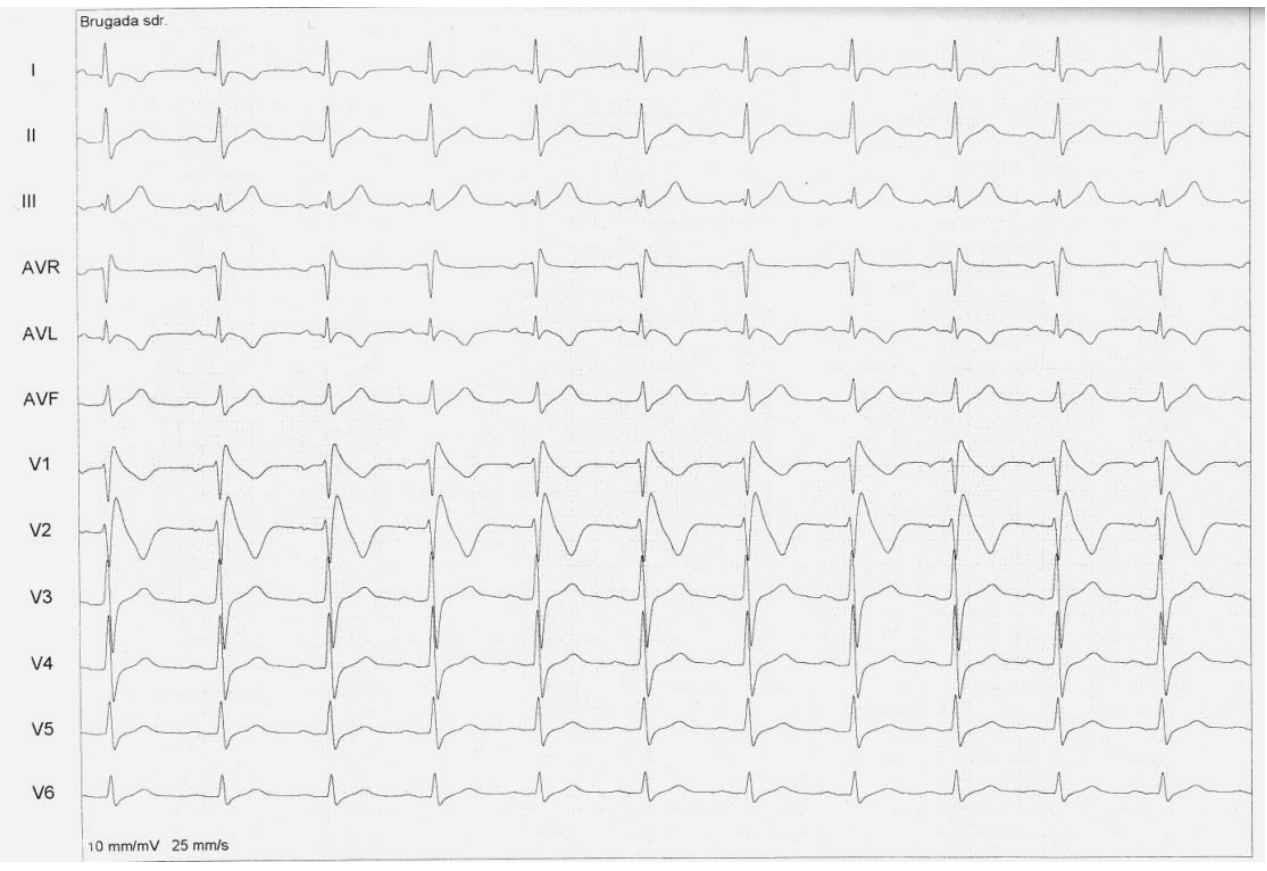

Fig. 3. 12 lead ECG documenting type 1 Brugada pattern during flecainide test

In less than 48 hours after implantation the device effectively made secondary prophylaxis by accurately detecting and reducing a VF episode with a $34.6 \mathrm{~J}$ shock (Figure 4). The patient was discharged home with appropriate follow-up instructions (a list of drugs to be avoided, recommendation for genetic screening of family members, regular ICD controls). 
Treated VT/VF Episode \#1

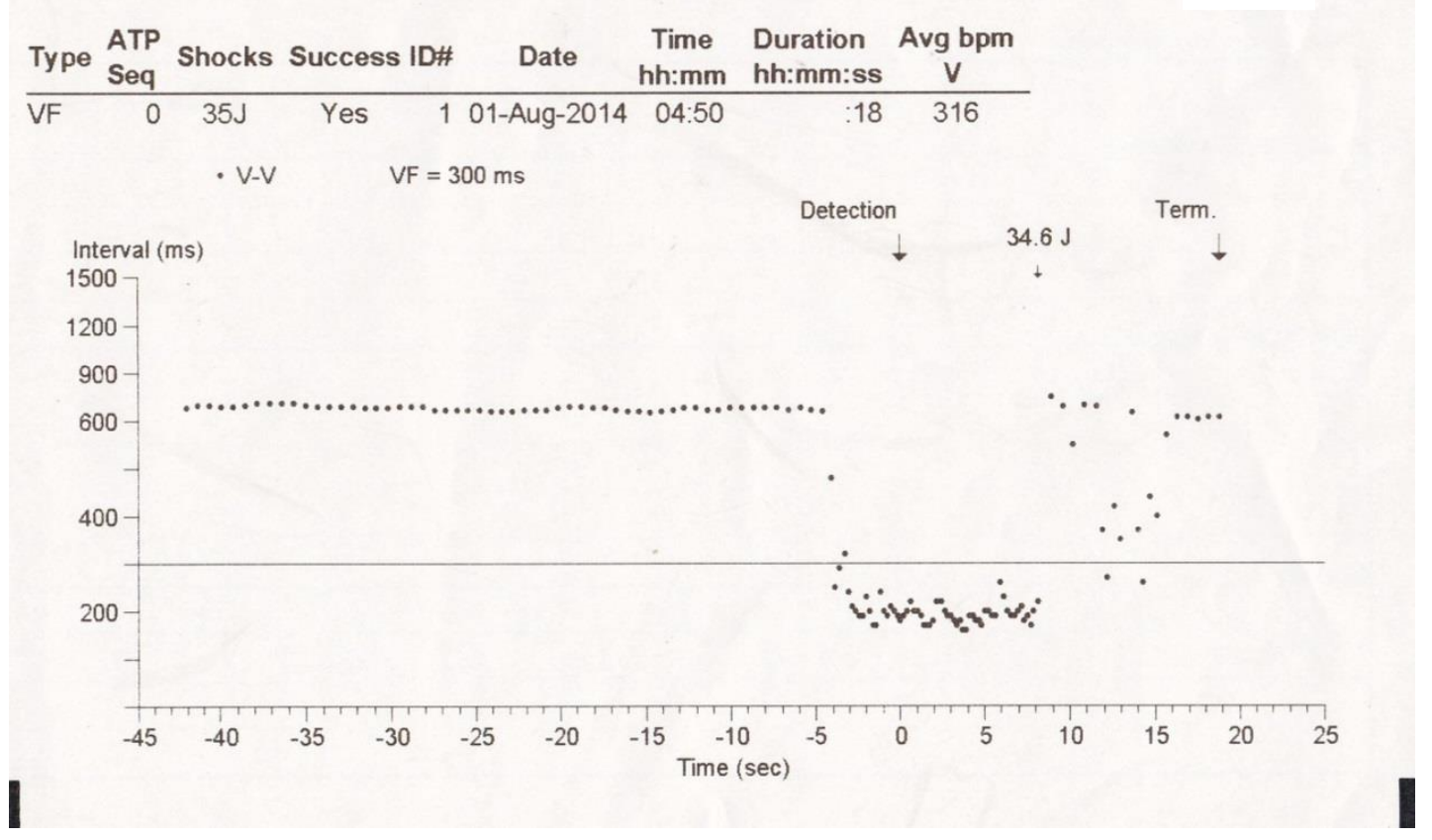

Fig. 4. Device interrogation - the ICD detected and reduced a VF episode

\section{Discussions}

BrS comprises extreme genetic heterogeneity, as at least 19 different genetic variants and more than 300 mutations have been reported so far $[3,8]$. The mutations are inherited in an autosomal dominant manner, with incomplete penetrance [9]. The dominant susceptibility gene is SCN5A, which encodes the alpha subunit of a myocardial sodium channel [10]. The overall prevalence of SCN5A mutations is $20-25 \%$, but SCN5A variants are even more frequent among children with BrS [11]. The phenotypic effect is disequilibrium between the natrium and potassium currents in the epicardial layers of the right ventricle $[3,8]$.

The clinical manifestations of Brugada syndrome generally appear in the third and fourth decade of life and are 10 times more frequent among male patients [8]. Some subjects experience palpitations, syncope, nocturnal agonal respiration or malignant ventricular arrhythmias. Ventricular fibrillation and sudden cardiac death commonly occur during nighttime, when the patient is resting. Supraventricular arrhythmias occur in $20 \%$ of BrS subjects: atrial fibrillation $(10-20 \%$ of cases), atrial flutter, atrioventricular nodal reentrant tachycardia, pre-excitation syndromes. [3, 8] The presence of supraventricular arrhythmias could be a sign of a more advanced condition, as they are more frequent among BrS patients who have an indication for ICD compared to those who do not require an implantable defibrillator $[3,8]$. Our patient experienced VF after strenuous physical exercise, a couple of days after a febrile episode. He also developed episodes of paroxysmal atrial fibrillation documented after the aborted SCD. Such clinical manifestations are valuable for risk stratification but are no longer included in the diagnostic criteria, since most subjects with Brugada electrocardiographic pattern never develop symptoms [8]. We considered the atrial fibrillation to be caused by the resuscitation maneuvers, since it was not documented on subsequent ECG recordings or the 24 hour Holter monitoring. We recommended annual screening for atrial fibrillation and decided that antiarrhythmic prophylaxis and anticoagulation are not necessary unless the arrhythmia reoccurs.

The hallmark of $\mathrm{BrS}$ is the presence of a ST segment elevation in at least one right 
precordial lead (V1-V3). Type 1 BrS appears as a coved elevation of at least $0.2 \mathrm{mV}$ in the $\mathrm{J}$ point, followed by a descending ST segment and a negative $T$ wave. This is the only diagnostic ECG aspect for BrS. Type II and III are suggestive for Brugada syndrome and appear as saddle-back shaped ST elevations, greater than $0.2 \mathrm{mV}$ for type II and less than $0.2 \mathrm{mV}$ for type III [3, 8].

The ECG pattern is persistent or intermittent and it can be unmasked by fever, vagal tone, sodium channel blockers and antidepressants [8]. Our patient presented a febrile episode 5 days prior to the aborted SCD, but he remained afebrile during hospitalization. ECG during fever should be performed whenever there is a suspicion of $\mathrm{BrS}$. The spontaneous presence of type I pattern is a strong predictor of arrhythmic episodes, but its presence can be documented in only $25 \%$ of tracings, even if a large number of ECGs are available for analysis. The diagnostic electrocardiographic aspect can be unmasked with the class IC antiarrhythmic drug test (ajmaline is considered the best option, particularly because of its short half time life) [3]. A negative test does not rule out the presence of a latent Brugada Syndrome, since a previous study showed that flecainide testing has $77 \%$ sensibility and $80 \%$ specificity [12]. The test should be performed in an electrophysiology laboratory as both ajmaline and flecainide can induce polymorphic ventricular tachycardia during infusion. Placing the V1 and V2 leads in the second intercostal spaces raises the specificity of ECG for exposing the BrS pattern, especially during a drug challenge [8]. Drugs acting on the sodium channel should be avoided in asymptomatic patients with Brugada ECG type I, II or III.

Electric shock can produce a Brugada-like electrocardiographic morphology [8], and this is the reason why we chose to perform the flecainide drug challenge even though our patient had already presented the diagnostic type 1 pattern.

Genetic testing provides the confirmation of the diagnosis, but is particularly useful in the screening of relatives at risk [8]. Although only $26 \%$ of subjects with documented $\mathrm{BrS}$ have a positive familial history of SCD, $36 \%$ of cases are diagnosed through familial screening [13]. We recommended the patient's grade I and II blood relatives to undergo genetic screening.

A previous study has shown that subjects with documented type I BrS have an annual risk of experiencing a cardiac event of $7.7 \%$ if they have had an aborted SCD. The risk drops to $1.9 \%$ if they had a history of syncope but remains $0.5 \%$ for asymptomatic individuals [13]. According to these results, our patient's annual risk was $7.7 \%$, but he experienced an episode of ventricular fibrillation just a couple of days after an aborted SCD.

Implantation of an ICD has been safely and effectively used for patients with type 1 Brugada pattern who have experienced documented malignant ventricular arrhythmias or cardio-respiratory arrest. The procedure can be performed in patients with type 1 morphology who are symptomatic (syncope) but programmed electrical stimulation (PES) inducible ventricular tachyarrhythmias are no longer an indication for implantable cardiac defibrillator $[8,14]$. Up to $30 \%$ of patients with implantable ICDs experience deviceassociated complications such as infection, inappropriate shocks and lead dysfunction during the first 10 years after the procedure [15]. Hence, ICDs are not indicated in asymptomatic subjects, who are known to have a very low arrhythmic risk [14]. Our patient was currently unemployed and was advised to avoid high-risk careers in which he could endanger his life or the life of others (professional driving, pilot, working at heights). The presence of the ICD will enable the subject to resume driving a month after the implant. It is recommended that ICDs are programmed with a single therapeutical window for ventricular fibrillation (in order to avoid inappropriate shocks), and we proceeded accordingly.

A recent study advocates that epicardial catheter ablation of the right ventricle outflow tract normalizes the ECG pattern and is effective in the prevention of ventricular arrhythmias, being especially useful in subjects with recurring electrical storms [16]. 


\section{Conclusions}

Brugada syndrome induces polymorphic ventricular tachycardia and ventricular fibrillation in the absence of structural heart disease. Implantable cardiac defibrillators have confirmed their effectiveness in preventing SCD in patients with symptomatic BrS. Further studies are needed in order to determine the appropriate therapeutic choice for asymptomatic subjects, especially those with PES-inducible ventricular tachyarrhythmias. In our case, the presence of previous cardiac arrest was an important risk marker for future cardiac events, which made our patient a candidate for secondary

\section{References}

1. Sovari AA, Prasun MA, Kocheril AG, Brugada R. Brugada syndrome unmasked by pneumonia. Tex Heart Inst J 2006; 33:501-504.

2. Brugada $P$, Brugada J. Right bundle branch block, persistent ST segment elevation and sudden cardiac death: a distinct clinical and electrocardiographic syndrome. A multicenter report. J Am Coll Cardiol 1992; 20:1391-1396.

3. Brugada $P$, Brugada $R$, Campuyzano $O$ et al. Brugada Syndrome 1992-2012: Twenty Years of Scientific Progress. In Zipes DP, Jalife J (Ed.) Cardiac Electrophysiology: From Cell to Bedside. Philadelphia: Elsevier Health Sciences; 2013:923-933.

4. Grogan SP, Cube RP, Edwards JA. Brugada syndrome unmasked by fever. Mil Med 2011; 176:946-949.

5. Noda T, Shimizu W, Tanaka K, Chayama K. Prominent $\mathrm{J}$ wave and ST segment elevation: serial electrocardiographic changes in accidental hypothermia. J Cardiovasc Electrophysiol 2003; 14:223.

6. Shimizu W. Acquired forms of the Brugada syndrome. J Electrocardiol 2005, 38(4):22-25.

7. Kranjcec D, Bergovec M, Rougier JS, et al. Brugada syndrome unmasked by accidental inhalation of gasoline vapors. Pacing Clin Electrophysiol 2007; 30:1294-1298.

8. Antzelevitch C, Patocskai B. Brugada Syndrome: Clinical, Genetic, Molecular, Cellular, and Ionic Aspects. Curr Probl Cardiol 2016; 41:7-57.

9. Wilde $A A$, Antzelevitch $C$, Borggrefe $M$, et al. Proposed diagnostic criteria for the Brugada syndrome: consensus report. Circulation 2002;106:2514-2519. prophylaxis. The patient suffered a second event 2 days after the procedure which was effectively detected and aborted by the ICD.

\section{List of abbreviations:}

BrS - Brugada Syndrome ECG - Electrocardiogram ICD - implantable cardioverter-defibrillator SCD - sudden cardiac death

VF - ventricular fibrillation

\section{Conflict of interest}

The authors declare that they have no competing interests.

10. Gourraud JB, Barc J, Thollet A, et al. The Brugada Syndrome: A Rare Arrhythmia Disorder with Complex Inheritance. Front Cardiovasc Med 2016; 3:9.

11. Andorin A, Behr ER, Denjoy I, et al. Impact of clinical and genetic findings on the management of young patients with Brugada syndrome. Heart Rhythm 2016; 13:1274-1282.

12. Meregalli $P G$, Ruijter JM, Hofman $N$, Bezzina CR, Wilde AA, Tan HL. Diagnostic value of flecainide testing in unmasking SCN5A-related Brugada syndrome. J Cardiovasc Electrophysiol 2006; 17:857-864.

13. Probst V, Veltmann C, Eckardt L, et al. Longterm prognosis of patients diagnosed with Brugada syndrome: Results from the FINGER Brugada Syndrome Registry. Circulation 2010; 121:635-643.

14. Priori SG, Blomstrom-Lundqvist $C$, Mazzanti A, et al. 2015 ESC Guidelines for the management of patients with ventricular arrhythmias and the prevention of sudden cardiac death: The Task Force for the Management of Patients with Ventricular Arrhythmias and the Prevention of Sudden Cardiac Death of the European Society of Cardiology (ESC). Endorsed by: Association for European Paediatric and Congenital Cardiology (AEPC). Eur Heart J 2015; 36:27932867.

15. Sacher F, Probst V, Maury $P$, et al. Outcome after implantation of a cardioverter-defibrillator in patients with Brugada syndrome: a multicenter study-part 2. Circulation 2013; 128:1739-1747. 
16. Nademanee K, Veerakul G, Chandanamattha $P$, et al. Prevention of ventricular fibrillation episodes in Brugada syndrome by catheter ablation over the anterior right ventricular outflow tract epicardium. Circulation 2011; 123:1270-1279. 